\title{
Avoiding Mode Pairing when Updating Finite Element Models
}

\author{
Magnus Andersson \\ Department of Electrical Engineering \\ Linköping University, S-581 83 Linköping, Sweden \\ www: http://www. control.isy.liu.se \\ email: magnusa@isy.liu.se \\ 1997-09-01
}

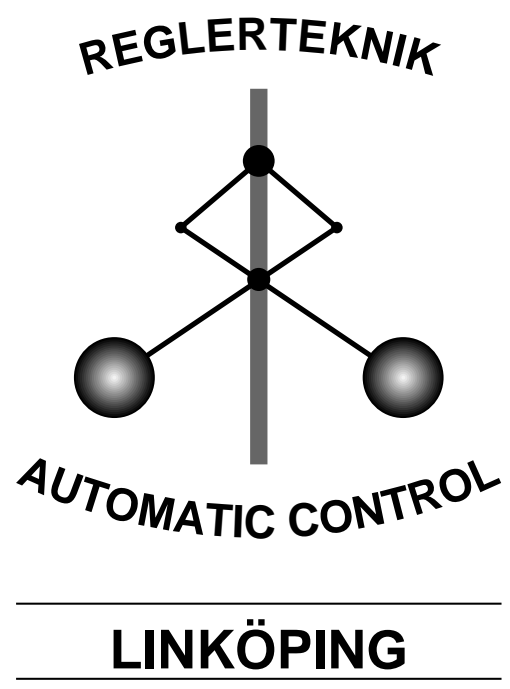

Technical reports from the Automatic Control group in Linköping are available as UNIX-compressed Postscript files by anonymous ftp at the address 130.236.20.24 (ftp.control.isy.liu.se). 


\title{
AVOIDING MODE PAIRING WHEN UPDATING FINITE ELEMENT MODELS
}

\author{
M. Andersson* T. Abrahamsson** \\ * Dept. of Electrical Engineering, Linköping University, Sweden, \\ E-mail: magnusa@isy.liu.se \\ ** SAAB AB, Linköping, Sweden, \\ E-mail: Thomas.Abrahamsson@saab.se
}

\begin{abstract}
Updating finite element models of complex mechanical structures requires some extra considerations. It is stressed that the two most important aspects on updating finite element models are parameter estimation properties and computational expenses. A novel mode-pairing free model updating formulation is found to have good parameter estimation properties. The computational expenses are reduced with a semi-fixed modal basis, kept fixed during several iterations.
\end{abstract}

Keywords: Parameter estimation; mechanical systems; nonlinear programming; model reduction.

\section{INTRODUCTION}

In aerospace applications it is for several reasons important that the FE model is accurate, i.e., that the model has a dynamical behavior close to the real structure. For instance, the security aspects on aero-planes require an experimental groundtest verification of the construction in a test setup. The verification includes measuring of the lowfrequency dynamical behavior of the structure for several configurations, e.g., for different amount of fuel in the wing-tanks. This measuring is very expensive and time-consuming. If an initial model of the structure can be updated/improved from the measurements of a single configuration, the other configurations can be simulated using the improved model. In order for these simulations to be valid, it is assumed that the improved model for the measured configuration is accurate, and that the differences between the configurations are easily accounted for in the model. This is mostly the case since the differences between the configurations are often known masses with known locations.
The specific task of improving an initial FE model using measurements is here denoted model updating. An attractive approach is to parameterize the FE model physically and estimate the numerical values of these parameters. This corresponds to the minimization of a cost function $V(\boldsymbol{\theta}) \geq 0$ describing the deviation between the model output and the measurements,

$$
\check{\boldsymbol{\theta}}=\underset{\boldsymbol{\theta} \in \boldsymbol{\Theta}}{\arg \min } V(\boldsymbol{\theta})
$$

where $\Theta$ denotes the open set of feasible values for the parameter vector $\boldsymbol{\theta}$. The minimization of $V$ in (1) is in general a non-linear problem. An iterative procedure is therefore required in order to obtain an estimate $\hat{\boldsymbol{\theta}}$ of $\check{\boldsymbol{\theta}}$,

$$
\boldsymbol{\theta}^{i+1}=\boldsymbol{\theta}^{i}+\boldsymbol{\Delta} \boldsymbol{\theta}^{i}, \boldsymbol{\theta}^{0}=\boldsymbol{\theta}^{i n i t}
$$

where

$$
\hat{\boldsymbol{\theta}}=\lim _{i \rightarrow \infty} \boldsymbol{\theta}^{i}
$$

Hopefully, a consistent estimate of the parameters are achievable, $\hat{\boldsymbol{\theta}}=\check{\boldsymbol{\theta}}$.

Updating FE models is a task of very high complexity. This is basically due to the size of the FE models and the high number of parameters $N_{\theta}$, 
but also the high number of actuators $m$ and sensors $p$ yielding a large amount of measurements. It is therefore important to reduce the computational expenses for model updating. For instance, model reduction is inevitable when dealing with FE models of complex industrial structures.

However, the complexity of the models is not the main obstacle for obtaining a successful updating. It is experimentally found that model updating formulations in general suffers from local minima in the parameter space. In order to alleviate the risk of ending up in a local minima, the convexity properties of the cost function describing the difference between the measurements and the model output must be enhanced.

\section{BASIC THEORY}

FE modeling of mechanical structures yield a mass matrix $\boldsymbol{M}$ and a stiffness matrix $\boldsymbol{K}$ of dimension $l \times l$, respectively. The normal modes $\phi_{i}$ and the resonance frequencies $\omega_{i}$ of the undamped $\mathrm{FE}$ model represented by $\boldsymbol{M}, \boldsymbol{K}$ are determined via the generalized eigenvalue problem

$$
\left(\boldsymbol{K}-\omega_{i}^{2} \boldsymbol{M}\right) \boldsymbol{\phi}_{i}=0, \boldsymbol{\phi}_{i}^{T} \boldsymbol{M} \boldsymbol{\phi}_{i}=1, i=1, \ldots, l
$$

where the normal modes $\phi_{i}$ are mass normalized. Notice that the modal matrix $\boldsymbol{\Phi}=\left[\phi_{1}, \ldots, \phi_{l}\right]$ diagonalizes $\boldsymbol{M}$ and $\boldsymbol{K}$ :

$$
\boldsymbol{\Phi}^{T} \boldsymbol{M} \boldsymbol{\Phi}=\boldsymbol{I}, \boldsymbol{\Phi}^{T} \boldsymbol{K} \boldsymbol{\Phi}=\operatorname{diag}\left(\omega_{1}^{2}, \ldots, \omega_{l}^{2}\right)=\mathbf{\Omega}^{2}
$$

where diag denotes a diagonal matrix. Two modes $\phi_{i}$ and $\phi_{j}$ are considered to be near multiple if their corresponding resonance frequencies $\omega_{i}$ and $\omega_{j}$ are close, i.e., if $\omega_{i} \approx \omega_{j}$.

A corresponding FE technique for modeling the damping is not available and ad hoc approaches to construct the damping matrix $\boldsymbol{L}$ is therefore inevitable. If $\boldsymbol{\Phi}$ also diagonalizes $\boldsymbol{L}$,

$$
\boldsymbol{\Phi}^{T} \boldsymbol{L} \boldsymbol{\Phi}=\operatorname{diag}\left(\gamma_{1}, \ldots, \gamma_{l}\right)=\boldsymbol{\Gamma}, \gamma_{i}=2 \xi_{i} \omega_{i}
$$

the damping is so-called proportional. In (6), the $\xi_{i}$ is the damping ratio of the normal mode $\phi_{i}$. The assumption of proportional damping is found to be valid for flexible structures in practice as well as in theory, see (Géradin and Rixen, 1994).

The input/output (I/O) behavior of a mechanical structure may be modeled

$$
\begin{aligned}
\boldsymbol{M} \ddot{\boldsymbol{x}}(t)+\boldsymbol{L} \dot{\boldsymbol{x}}(t)+\boldsymbol{K} \boldsymbol{x}(t) & =\boldsymbol{B} \boldsymbol{u}(t) \\
\boldsymbol{y}(t) & =\boldsymbol{C} \boldsymbol{x}(t)
\end{aligned}
$$

where $\boldsymbol{x}$ is the vector of the $\boldsymbol{l}$ degrees of freedom (dof.), $\boldsymbol{u}$ is the vector of $m$ input forces, and $\boldsymbol{y}$ the vector of $p$ displacement measurements. In (7), $\boldsymbol{B}$ (of dimension $l \times m$ ) describes the positions of the actuators yielding the input forces and $C(p \times l)$ the positions of the sensors measuring the output. Equivalently, (7) may be written

$$
\begin{aligned}
\ddot{\boldsymbol{q}}(t)+\boldsymbol{\Gamma} \dot{\boldsymbol{q}}(t)+\boldsymbol{\Omega}^{2} \boldsymbol{q}(t) & =\boldsymbol{\Phi}^{T} \boldsymbol{B} \boldsymbol{u}(t) \\
\boldsymbol{y}(t) & =\boldsymbol{C} \boldsymbol{\Phi}(t)
\end{aligned}
$$

where $\boldsymbol{q}=\boldsymbol{\Phi} \boldsymbol{x}$ is a transformation to the modal coordinates $\boldsymbol{q}$.

The frequency response function (FRF) of (7) is defined as the transfer function $\boldsymbol{G}(s)$ evaluated on the imaginary axis, $s=j \omega$,

$$
\boldsymbol{G}(\omega)=\boldsymbol{C}\left(\boldsymbol{K}+j \omega \boldsymbol{L}-\omega^{2} \boldsymbol{M}\right)^{-1} \boldsymbol{B}
$$

where $\omega$ denotes frequency, and where $\boldsymbol{G}(\omega)$ denotes $\boldsymbol{G}(j \omega)$ for simplicity. The FRF of (8) may be conveniently written as

$$
\boldsymbol{G}(\omega)=\sum_{i=1}^{l} \frac{\boldsymbol{C} \phi_{i} \boldsymbol{\phi}_{i}^{T} \boldsymbol{B}}{\omega_{i}^{2}-\omega^{2}+j 2 \xi_{i} \omega_{i} \omega}
$$

showing the interpretation of the triple $\left\{\phi_{i}, \xi_{i}, \omega_{i}\right\}$ of modal parameters in the frequency domain.

An efficient reduction of the system matrices $\boldsymbol{M}, \boldsymbol{L}, \boldsymbol{K}$ to the low-frequency area can be performed as

$$
\boldsymbol{M}_{r}=\boldsymbol{\Phi}_{r}^{T} \boldsymbol{M} \boldsymbol{\Phi}_{r}, \boldsymbol{L}_{r}=\boldsymbol{\Phi}_{r}^{T} \boldsymbol{L} \boldsymbol{\Phi}_{r}, \boldsymbol{K}_{r}=\boldsymbol{\Phi}_{r}^{T} \boldsymbol{K} \boldsymbol{\Phi}_{r}
$$

using a reduced modal matrix $\Phi_{r}=\left[\phi_{i_{1}}, \ldots, \phi_{i_{r}}\right]$. It remains to determine the $i_{j}$ 's. Traditionally, $\phi_{i_{j}}$ are chosen through a pairing of the normal modes $\phi_{i}$ of the model and the corresponding estimates of the structure $\hat{\phi}_{j}^{x}$,

$$
\phi_{i_{j}} \leftrightarrow \hat{\phi}_{j}^{x}, j=1, \ldots, N_{q}^{x}
$$

where $N_{q}^{x}$ denotes the number of estimated modes of the structure. The actual pairing of modes often relies on the correlation between the $\phi_{i}$ and the $\hat{\phi}_{j}^{x}$. The pairing of modes also implies a pairing of the corresponding triples of modal parameters,

$$
\left\{\phi_{i_{j}}, \xi_{i_{j}}, \omega_{i_{j}}\right\} \leftrightarrow\left\{\hat{\phi}_{j}^{x}, \hat{\xi}_{j}^{x}, \hat{\omega}_{j}^{x}\right\}, j=1, \ldots, N_{q}^{x}
$$

giving the possibility to construct a reduced damping matrix $\boldsymbol{L}_{r}$, and incorporating deviation in the resonance frequencies in the cost function.

The mode-pairing in (12) is a combinatorial optimization problem of rather high complexity. For large flexible structures with many modes in the model/structure, sub-optimal algorithms must be used in order to decrease the time for the modepairing.

The estimate $\hat{\boldsymbol{G}}^{x}$ of $\boldsymbol{G}^{x}$ at the frequencies $\omega_{R, i}, i=1, \ldots, N_{\omega}$ can be obtained as

$$
\hat{G}_{k l}\left(\omega_{R, i}\right)=\frac{y_{k}\left(\omega_{R, i}\right)}{u_{l}\left(\omega_{R, i}\right)}
$$

where $\hat{G}_{k l}$ is the entry of row $k$ and column $l$ of $\hat{\boldsymbol{G}}^{x}$. In $(14), y_{k}\left(\omega_{R, i}\right)$ denotes the Fourier 
transform of the output from sensor $k$, and where $u_{l}\left(\omega_{R, i}\right)$ denotes the Fourier transform of the sinusoidal input force applied with actuator $l$.

In (Williams et al., 1985), the multi-variate mode indicator function (MMIF) is presented. It is used to detect (multiple) modes of the structure as well as compute an input force vector $\boldsymbol{U}_{i}$. The input signal $\boldsymbol{u}(t)=\boldsymbol{U}_{i} \sin \left(\hat{\omega}_{i}^{x} t\right)$ is then applied to the structure in order to obtain the estimate $\hat{\phi}_{i}^{x}$,

$$
\hat{\boldsymbol{\phi}}_{i}^{x}=\operatorname{Im}\left\{\boldsymbol{G}^{x}\left(\hat{\omega}_{i}^{x}\right) \boldsymbol{U}_{i}\right\}
$$

for $i=1, \ldots, N_{q}^{x}$, see (Andersson, 1997). The $\boldsymbol{U}_{i}$ is the eigenvector $\lambda_{i, \min }$

$$
\lambda_{i, \min }=\min _{j} \lambda_{i, j}, i=1, \ldots, N_{q}^{x}
$$

where

$$
\left(\boldsymbol{A}_{i}-\lambda_{i, j}\left(\boldsymbol{A}_{i}+\boldsymbol{B}_{i}\right)\right) \boldsymbol{U}_{i, j}=0, j=1, \ldots, m
$$

where $\boldsymbol{A}_{i}=\operatorname{Re}\left\{\hat{\boldsymbol{G}}^{x}\left(\hat{\omega}_{i}^{x}\right)\right\}^{T} \operatorname{Re}\left\{\hat{\boldsymbol{G}}^{x}\left(\hat{\omega}_{i}^{x}\right)\right\}$ and $\boldsymbol{B}_{i}=\operatorname{Im}\left\{\hat{\boldsymbol{G}}^{x}\left(\hat{\omega}_{i}^{x}\right)\right\}^{T} \operatorname{Im}\left\{\hat{\boldsymbol{G}}^{x}\left(\hat{\omega}_{i}^{x}\right)\right\}$.

\section{MODEL UPDATING FORMULATION}

In ground vibration testing, the estimate $\hat{\boldsymbol{G}}^{x}$ of $G^{x}$ is obtained in the low-frequency area. The estimated triple of modal parameters $\left\{\hat{\phi}_{i}^{x}, \hat{\xi}_{i}^{x}, \hat{\omega}_{i}^{x}\right\}$ are also commonly available.

It is experimentally found that the previous described mode-pairing technique may introduce severe local minima in the parameter space of the cost function $V$. In particular, the assumption of a valid mode pairing is critical whenever the dynamical behavior of the model is not close to the structure's, i.e., when $\boldsymbol{\theta}^{i} \not \check{\boldsymbol{\theta}}$, or when near multiple modes of the model and/or the structure are present. An objective have therefore been to develop a mode-pairing free technique to update FE models of complex mechanical structures.

It is also found that a combined modal/frequency domain cost function based on both deviations in resonance frequencies and deviations in properties defined by the the FRF has good parameter estimate properties (Andersson, 1997), i.e., an estimate $\hat{\boldsymbol{\theta}}$ of $\check{\boldsymbol{\theta}}$ where

$$
\hat{\boldsymbol{\theta}} \approx \check{\boldsymbol{\theta}}, V(\hat{\boldsymbol{\theta}}) \ll V\left(\boldsymbol{\theta}^{0}\right)
$$

where $\boldsymbol{\theta}^{0}$ is the initial value of the parameter vector $\boldsymbol{\theta}$.

\subsection{Model reduction}

Defining $\hat{\phi}_{i}$ as

$$
\hat{\boldsymbol{\phi}}_{i}=\boldsymbol{\Phi}_{q} \hat{\boldsymbol{a}}_{i}, i=1, \ldots, N_{q}^{x}
$$

where

$$
\begin{gathered}
\boldsymbol{\Phi}_{q}=\left[\boldsymbol{\phi}_{1}, \ldots, \boldsymbol{\phi}_{N_{q}}\right] \\
\hat{\boldsymbol{a}}_{i}=\underset{\boldsymbol{a} \in \mathbb{R}^{N_{q}}}{\arg \min }\left\|\boldsymbol{\Phi}_{q} \boldsymbol{a}_{i}-\hat{\boldsymbol{\phi}}_{i}^{x}\right\|_{2}
\end{gathered}
$$

where $N_{q}$ denotes the number of computed normal modes. The matrix of reduction $\bar{\Phi}_{r}$ is then defined as

$$
\overline{\mathbf{\Phi}}_{r}=\left[\bar{\phi}_{i}, \ldots, \bar{\phi}_{N_{q}^{x}}\right], \bar{\phi}_{i}=\frac{1}{\left(\left(\hat{\phi}_{i}\right)^{T} \boldsymbol{M} \hat{\phi}_{i}\right)^{1 / 2}} \hat{\boldsymbol{\phi}}_{i}
$$

where $\bar{\phi}_{i}$ is the mass-normalized version of $\hat{\phi}_{i}$. Notice that the mass-normalization of $\hat{\phi}_{i}$ in (22) is based on the assumption that the mass matrix $\boldsymbol{M}$ of the model is approximating the mass distribution of the structure sufficiently accurate.

The reduced system matrices $\overline{\boldsymbol{M}}_{r}$ and $\overline{\boldsymbol{K}}_{r}$ become

$$
\overline{\boldsymbol{M}}_{r}=\overline{\boldsymbol{\Phi}}_{r}^{T} \boldsymbol{M} \overline{\boldsymbol{\Phi}}_{r}, \overline{\boldsymbol{K}}_{r}=\overline{\boldsymbol{\Phi}}_{r}^{T} \boldsymbol{K} \overline{\boldsymbol{\Phi}}_{r}
$$

\subsection{Damping model design}

In order to obtain a reduced damping matrix $\overline{\boldsymbol{L}}_{r}$, some assumptions about the damping distribution in the structure must be made. Let $\boldsymbol{\Phi}_{x}=\left[\phi_{1}^{x}, \ldots, \phi_{N_{q}^{x}}^{x}\right]$ denote the "true" modes of the structure, and let $\boldsymbol{L}_{x}$ denote the "true" damping matrix of the structure. If the damping distribution in the structure are proportional, then $\boldsymbol{\Phi}_{x}^{T} \boldsymbol{L}_{x} \boldsymbol{\Phi}_{x}$ will be diagonal. Assuming that $\hat{\boldsymbol{\phi}}_{i}^{x}$ is parallel with $\phi_{i}^{x}$, and that $\bar{\phi}_{i}$ is parallel with $\hat{\phi}_{i}^{x}$ :

$$
\hat{\phi}_{i}^{x} \| \phi_{i}^{x}, \text { and } \hat{\phi}_{i}^{r} \| \hat{\phi}_{i}^{x}, i=1, \ldots, N_{q}^{x}
$$

then also $\overline{\boldsymbol{\Phi}}_{r}^{T} \boldsymbol{L}_{x} \overline{\boldsymbol{\Phi}}_{r}$ will be diagonal. These assumptions indicate that $\overline{\boldsymbol{L}}_{r}$ may be modeled as

$$
\overline{\boldsymbol{L}}_{r}=\operatorname{diag}\left(\ldots, 2 \hat{\xi}_{i}^{x} \bar{\omega}_{i}, \ldots\right)
$$

where $\bar{\omega}_{i}$ is the square root of the Rayleigh quotient $R$ in

$$
R\left(\bar{\phi}_{i}\right)=\frac{\bar{\phi}_{i}^{T} \boldsymbol{K} \bar{\phi}_{i}}{\bar{\phi}_{i}^{T} \boldsymbol{M} \bar{\phi}_{i}}=\left(\bar{\omega}_{i}\right)^{2}, i=1, \ldots, N_{q}^{x}
$$

see (Craig, Jr, 1981).

However, the matrices $\overline{\boldsymbol{M}}_{r}$ and $\overline{\boldsymbol{K}}_{r}$ are in general not diagonal, i.e., the damping is not proportional since $\overline{\boldsymbol{L}}_{r}$ is diagonal. If a proportional damping is required during the model updating, a diagonalization of $\overline{\boldsymbol{M}}_{r}$ and $\overline{\boldsymbol{K}}_{r}$ can first be made as

$$
\overline{\boldsymbol{M}}_{r}^{d}=\boldsymbol{\Psi}^{T} \overline{\boldsymbol{M}}_{r} \boldsymbol{\Psi}, \overline{\boldsymbol{K}}_{r}^{d}=\boldsymbol{\Psi}^{T} \overline{\boldsymbol{K}}_{r} \boldsymbol{\Psi}
$$

where

$$
\boldsymbol{\Psi}=\left[\boldsymbol{\psi}_{1}, \ldots, \boldsymbol{\psi}_{N_{q}^{x}}\right]
$$

In (28), the basis vectors $\boldsymbol{\psi}_{i}$ is defined by

$$
\left(\overline{\boldsymbol{K}}_{r}-\varpi_{i}^{2} \overline{\boldsymbol{M}}_{r}\right) \boldsymbol{\psi}_{i}=0, \boldsymbol{\psi}_{i}^{T} \overline{\boldsymbol{M}}_{r} \boldsymbol{\psi}_{i}=1
$$


for $i=1, \ldots, N_{q}^{x}$, compare with (4)-(5). The proportional damping matrix $\overline{\boldsymbol{L}}_{r}^{d}$ may be approximated by the diagonal of $\boldsymbol{\Psi}^{T} \overline{\boldsymbol{L}}_{r} \boldsymbol{\Psi}$ :

$$
\overline{\boldsymbol{L}}_{r}^{d}=\operatorname{diag}\left(\boldsymbol{\Psi}^{T} \overline{\boldsymbol{L}}_{r} \boldsymbol{\Psi}\right)=\operatorname{diag}\left(\ldots, \bar{\zeta}_{i}, \ldots\right)
$$

Notice that the damping of the updated model becomes proportional if the parameter estimation is consistent $(\hat{\boldsymbol{\theta}}=\check{\boldsymbol{\theta}})$, and if the mode estimates are unbiased, i.e., if $\hat{\phi}_{i}^{x} \| \phi_{i}^{x}$. This is true even if non-proportional damping is used during the model updating.

The reduced model (for proportional damping) becomes

$$
\begin{aligned}
\overline{\boldsymbol{M}}_{r}^{d} \ddot{\boldsymbol{q}}+\overline{\boldsymbol{L}}_{r}^{d} \dot{\boldsymbol{q}}+\overline{\boldsymbol{K}}_{r}^{d} \boldsymbol{q} & =\left(\overline{\boldsymbol{\Phi}}_{r}^{d}\right)^{T} \boldsymbol{B} \boldsymbol{u} \\
y & =\boldsymbol{C} \overline{\boldsymbol{\Phi}}_{r}^{d} \boldsymbol{q}
\end{aligned}
$$

where

$$
\overline{\mathbf{\Phi}}_{r}^{d}=\overline{\mathbf{\Phi}}_{r} \boldsymbol{\Psi}
$$

The FRF corresponding to (31) becomes

$$
\overline{\boldsymbol{G}}_{r}^{d}(\omega)=\boldsymbol{C} \overline{\boldsymbol{\Phi}}_{r}^{d}\left(\overline{\boldsymbol{Z}}_{r}^{d}(\omega)\right)^{-1}\left(\overline{\boldsymbol{\Phi}}_{r}^{d}\right)^{T} \boldsymbol{B}
$$

where

$$
\overline{\boldsymbol{Z}}_{r}^{d}(\omega)=\operatorname{diag}\left(\ldots, \varpi_{i}^{2}-\omega^{2}+j \bar{\zeta}_{i} \omega, \ldots\right)
$$

\subsection{Cost function}

Using all the asssumed measurements avilable, a mode-pairing free cost function can be obtained as

$$
V(\boldsymbol{\theta})=\nu_{1}^{2} V_{1}(\boldsymbol{\theta})+\nu_{2}^{2} V_{2}(\boldsymbol{\theta})+\nu_{3}^{2} V_{3}(\boldsymbol{\theta})
$$

where $\nu_{i}$ are cost function weights, and where

$$
\begin{aligned}
& V_{1}(\boldsymbol{\theta})=\sum_{i=1}^{N_{\omega}}\left\|\overline{\boldsymbol{G}}_{r}^{d}\left(\boldsymbol{\theta}, \omega_{R, i}^{x}\right)-\hat{\boldsymbol{G}}^{x}\left(\omega_{R, i}^{x}\right)\right\|_{F}^{2} \\
& V_{2}(\boldsymbol{\theta})=\sum_{i=1}^{N_{q}^{x}}\left|\frac{\bar{\omega}_{i}(\boldsymbol{\theta})-\hat{\omega}_{i}^{x}}{\hat{\omega}_{i}^{x}}\right|_{2}^{2} \\
& V_{3}(\boldsymbol{\theta})=\sum_{i=1}^{N_{q}^{x}}\left\|\overline{\boldsymbol{G}}_{r}^{d}\left(\boldsymbol{\theta}, \hat{\omega}_{i}^{x}\right) \boldsymbol{U}_{i}^{x}-\boldsymbol{Y}_{i}^{x}\right\|_{2}^{2}
\end{aligned}
$$

where $F$ denotes the Frobenius norm, and $\omega_{R, i}^{x}$ denotes the measured frequency points of $\hat{\boldsymbol{G}}^{x}$. Minimizing $V_{1}$ corresponds to the maximum-likelihood (ML) estimate of $\check{\boldsymbol{\theta}}$, see (McKelvey, 1995). The $V_{2}$ is included in order to increase the convexity properties of the cost function, see (Abrahamsson et al., 1996) where the enhanced parameter estimation properties using the Rayleigh quotient in the cost function are studied. In $(38), \boldsymbol{Y}_{i}^{x}$ corresponds to the output from the structure for the input $\boldsymbol{u}(t)=\boldsymbol{U}_{i}^{x} \sin \left(\hat{\omega}_{i}^{x} t\right)$

$$
\boldsymbol{Y}_{i}^{x}=\boldsymbol{G}^{x}\left(\hat{\omega}_{i}^{x}\right) \boldsymbol{U}_{i}^{x}
$$

\subsection{Implementation}

Using numerical difference approximations of $\partial \boldsymbol{M} / \partial \boldsymbol{\theta}$ and $\partial \boldsymbol{K} / \partial \boldsymbol{\theta}$, approximating derivatives of $\overline{\boldsymbol{M}}_{r}, \overline{\boldsymbol{K}}_{r}$ are obtained as

$$
\begin{aligned}
\frac{\partial \overline{\boldsymbol{M}}_{r}}{\partial \theta_{k}} & \approx \overline{\boldsymbol{\Phi}}_{r}^{T} \frac{\boldsymbol{M}\left(\theta_{k}+\delta \theta_{k}\right)-\boldsymbol{M}\left(\theta_{k}\right)}{\delta \theta_{k}} \overline{\boldsymbol{\Phi}}_{r} \\
\frac{\partial \overline{\boldsymbol{K}}_{r}}{\partial \theta_{k}} & \approx \overline{\boldsymbol{\Phi}}_{r}^{T} \frac{\boldsymbol{K}\left(\theta_{k}+\delta \theta_{k}\right)-\boldsymbol{K}\left(\theta_{k}\right)}{\delta \theta_{k}} \overline{\boldsymbol{\Phi}}_{r}
\end{aligned}
$$

If the modal basis of reduction $\overline{\boldsymbol{\Phi}}_{r}$ and the linearization of $\overline{\boldsymbol{M}}_{r}$ and $\overline{\boldsymbol{K}}_{r}$,

$$
\begin{aligned}
\overline{\boldsymbol{M}}_{r}^{l i n}(\boldsymbol{\theta}+\boldsymbol{\Delta} \boldsymbol{\theta}) & \approx \overline{\boldsymbol{M}}_{r}(\boldsymbol{\theta})+\sum_{k} \frac{\partial \overline{\boldsymbol{M}}_{r}(\boldsymbol{\theta})}{\partial \boldsymbol{\theta}_{k}} \Delta \theta_{k} \\
\overline{\boldsymbol{K}}_{r}^{l i n}(\boldsymbol{\theta}+\boldsymbol{\Delta} \boldsymbol{\theta}) & \approx \overline{\boldsymbol{K}}_{r}(\boldsymbol{\theta})+\sum_{k} \frac{\partial \overline{\boldsymbol{K}}_{r}(\boldsymbol{\theta})}{\partial \boldsymbol{\theta}_{k}} \Delta \theta_{k}
\end{aligned}
$$

are semi-fixed, i.e., kept fixed for several iterations, the computational expenses can be reduced, see (Andersson, 1997).

In Fig. 1, a flow-chart of a general model updating strategy is shown.

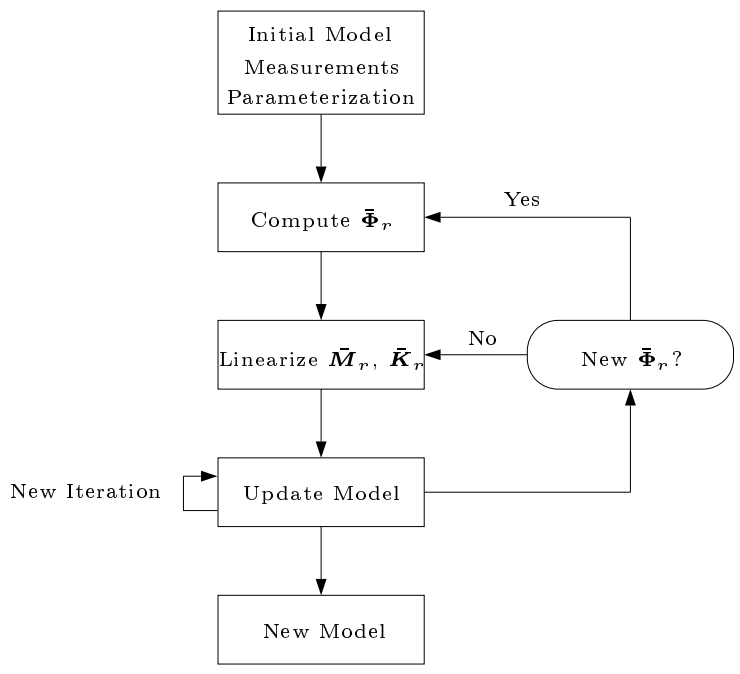

Fig. 1. A flow chart of the general model updating procedure.

Let $V(\boldsymbol{\theta})=\boldsymbol{v}(\boldsymbol{\theta})^{T} \boldsymbol{v}(\boldsymbol{\theta})$ where $\boldsymbol{v}(\boldsymbol{\theta})=\boldsymbol{v}_{m}(\boldsymbol{\theta})-\boldsymbol{v}_{s}$ is the deviation vector between the output of the model $\boldsymbol{v}_{m}$ and the measurements of the structure $\boldsymbol{v}_{s}$. Linearizing $\boldsymbol{v}$ in $\boldsymbol{\theta}$,

$$
\boldsymbol{v}(\boldsymbol{\theta}+\boldsymbol{\delta} \boldsymbol{\theta}) \approx \boldsymbol{v}(\boldsymbol{\theta})+\boldsymbol{J}(\boldsymbol{\theta}) \boldsymbol{\delta} \boldsymbol{\theta}
$$

the parameter difference vector $\boldsymbol{\Delta} \boldsymbol{\theta}^{i}$ in (2) can be determined as

$$
\begin{gathered}
\Delta \boldsymbol{\theta}^{i}=\underset{\boldsymbol{\Delta} \boldsymbol{\theta}}{\arg \min }\left\{\left\|\boldsymbol{J}\left(\boldsymbol{\theta}^{i}\right) \boldsymbol{\Delta} \boldsymbol{\theta}-\boldsymbol{d}\left(\boldsymbol{\theta}^{i}\right)\right\|_{2}^{2}\right. \\
\left.+\mu^{2}\|\boldsymbol{\Delta} \boldsymbol{\theta}\|_{2}^{2}\right\}
\end{gathered}
$$

where $\boldsymbol{d}\left(\boldsymbol{\theta}^{i}\right)=\boldsymbol{v}_{m}\left(\boldsymbol{\theta}^{i}\right)-\boldsymbol{v}_{s}$, and $\mu$ denotes the regularization term, see (Moré, 1983). In 
(Andersson, 1997), a robust strategy for determining the value of $\mu$ is proposed. It is a modified version of Fletcher's strategy in (Wolfe, 1978), being more robust against ending up in undesired local minima.

\section{NUMERICAL SIMULATION EXAMPLE}

Fig. 2 shows a simple aircraft-like structure.

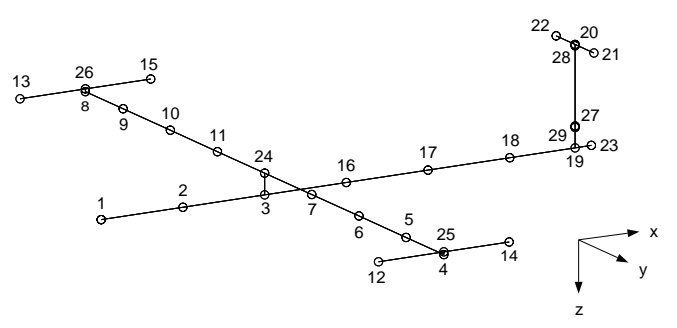

Fig. 2. The Garteur model.

In the examples below, 30 sensors are used to collect measurements. The cost function weights $\nu_{i}$ are determined so that

$$
V_{1}=\frac{10}{14} V, V_{2}=\frac{1}{14} V, V_{3}=\frac{3}{14} V
$$

in (36)-(38) for the initial values of the parameters, $\boldsymbol{\theta}=\boldsymbol{\theta}^{\text {init }}$.

\subsection{Example 1}

If the actuators are located to $\left\{12_{z}, 14_{x}, 15_{z}, 20_{y}\right\}$, the 9 low-frequency modes are estimated appropriately, since

$$
\min _{i} \frac{\left|\left(\phi_{i}^{x}\right)^{T} \hat{\phi}_{i}^{x}\right|^{2}}{\left|\phi_{i}^{x}\right|^{2}\left|\hat{\phi}_{i}^{x}\right|^{2}}=0.99
$$

The parameter history then become as in Fig. 3. It is seen that the estimated parameter values $\hat{\boldsymbol{\theta}}$ have converged to the global minima in 13 iterations. The cost function history is shown in Fig. 4. It is seen that the linearization $\overline{\boldsymbol{M}}_{r}^{\text {lin }}, \overline{\boldsymbol{K}}_{r}^{\text {lin }}$ is valid since the circles (o) is close to the solid line. The * denotes the values of the cost function for a new computation of the modal basis of reduction $\overline{\mathbf{\Phi}}_{r}$.

\subsection{Example 2}

If the actuators are located to $\left\{12_{z}, 15_{z}, 20_{y}\right\}$, the 9 low-frequency modes are not estimated appropriately, since

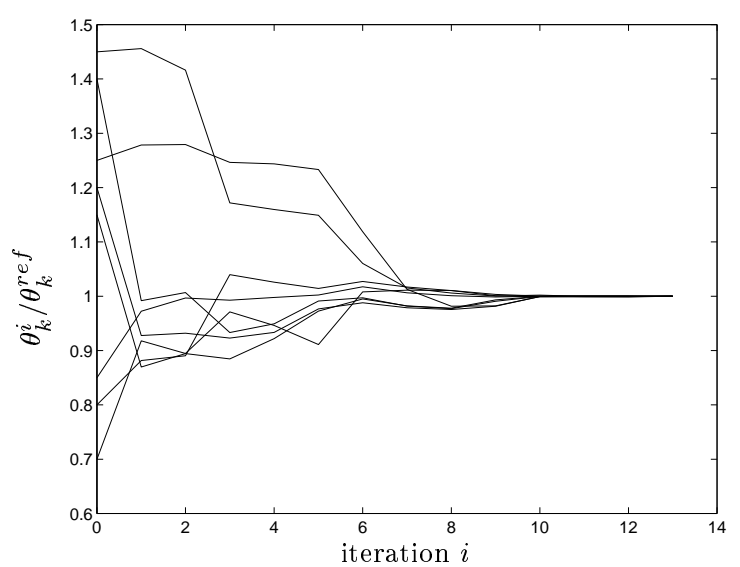

Fig. 3. The history of the relative parameter values, Example 1.

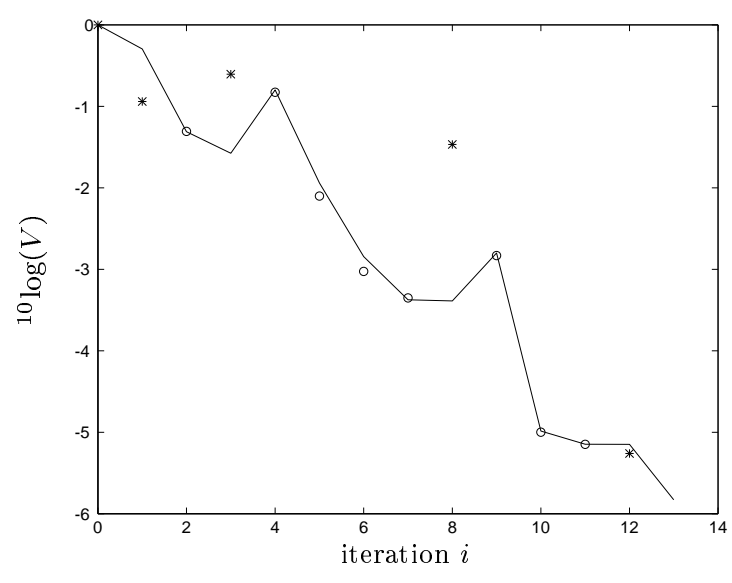

Fig. 4. The history of the cost function, Example 1.

$$
\min _{i} \frac{\left|\left(\phi_{i}^{x}\right)^{T} \hat{\phi}_{i}^{x}\right|^{2}}{\left|\phi_{i}^{x}\right|^{2}\left|\hat{\phi}_{i}^{x}\right|^{2}}=0.87
$$

The parameter history becomes as in Fig. 5. It can be stated that not appropriately estimated modes may turn up as bias in the parameter estimate.

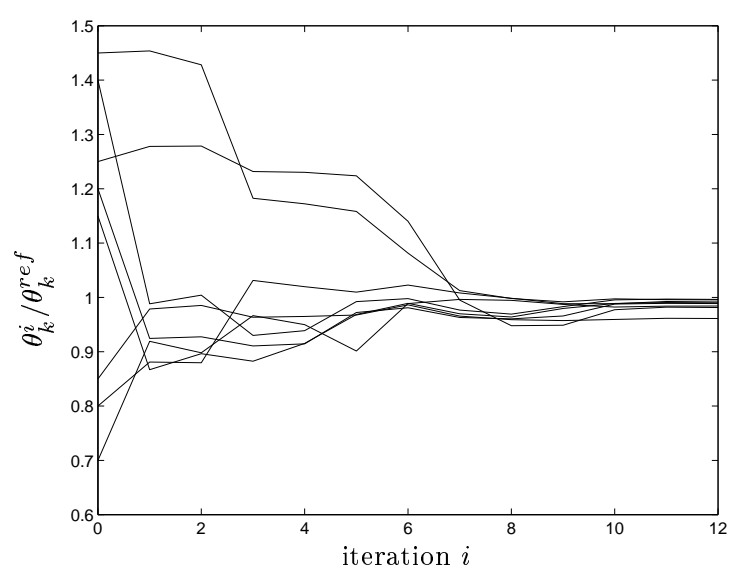

Fig. 5. The history of the relative parameter values, Example 2. 
However, even if the mode estimates $\hat{\phi}_{i}^{x}$ are not parallel with the true modes of the structure $\phi_{i}^{x}$, the cost function decrease is substantial:

$$
V(\hat{\boldsymbol{\theta}})=6.7 \cdot 10^{-5} \cdot V\left(\boldsymbol{\theta}^{\text {init }}\right)
$$

The relative large deviation in the estimated parameter values $\hat{\boldsymbol{\theta}}$ from $\check{\boldsymbol{\theta}}$ is in view of (50) due to that the parameters are dependent, i.e., the deviation in a parameter value is compensated by the deviations in the other parameter values.

\section{CONCLUSIONS}

It was pointed out that the two most important aspects on a model updating formulation is its parameter estimation properties and its computational complexity. It was also noted that model updating in general suffers from undesired local minima in the cost function. In order to reduce the risk of ending up in a local minima, the traditional technique of pairing modes was circumvented when constructing the reduced size model. A novel combined frequency domain/modal domain mode-pairing free cost function was in simulation examples shown to yield good parameter estimates if the modes of structure are appropriately estimated. If the modes of the structure are not appropriately estimated, bias in the parameter estimates emerged. The value of the cost function are reduced considerably, though. A modal reduction basis was used in several iterations in order to reduce the time-consuming computation of the normal modes.

\section{REFERENCES}

Abrahamsson, T., M. Andersson and T. McKelvey (1996). A finite element model updating formulation using frequency responses and eigenfrequencies. In: Second International Conference on Structural Dynamics Modelling - Test, Analysis and Correlation. DTA/NAFEMS. pp. 293-305.

Andersson, M. (1997). Experimental design and updating of finite element models. Lic. thesis. Automatic Control, Dept. of EE, Linköping University, 58183 Linköping, Sweden.

Géradin, M. and D. Rixen (1994). Mechanical Vibrations - Theory and Application to Structural Dynamics. Wiley.

Craig, Jr, R. R. (1981). Structural Dynamics. An Introduction to Computer Methods. Wiley.

McKelvey, T. (1995). Identification of State-Space Models from Time and Frequency Data. PhD thesis. Linköping University. Dept. of Electr. Eng., Linköping University, 58183 Linköping.

Moré, J. J. (1983). Recent developments inalgorithms and software for trust region methods.
In: Mathematical Programming. The State of the Art (A. Bachem, M. Grötchel and B. Korte, Eds.). Springer-Verlag. pp. 258-287.

Williams, R., J. Crowley and H. Vold (1985). The multivariate mode indicator function in modal analysis. In: Proceedings of the 3rd International Modal Analysis Conference. pp. 66-70.

Wolfe, M. A. (1978). Numerical Methods for Unconstrained Optimization-an introduction. Van Nostrand Reinhold. 